\title{
G7-Finanzgipfel in Dresden
}

Drei Fragen standen auf der Tagesordnung des G7-Finanzgipfels der Finanzminister und Notenbankchefs in Dresden: Wie kann die Weltwirtschaft zu mehr Wirtschaftswachstum kommen? Ist die Finanzmarktregulierung so reformiert worden, dass Finanzkrisen künftig verhindert werden können? Was ist zu tun, um Steuervermeidung durch multinationale Unternehmen einzudämmen?

Bei der ersten Frage ging es darum, die Gräben zwischen Befürwortern und Gegnern schuldenfinanzierter staatlicher Konjunkturprogramme zu überwinden. Vor allem Deutschland wird von seinen Partnerländern seit vielen Jahren immer wieder aufgefordert, mehr Schulden aufzunehmen, um die Konjunktur zu stimulieren. In Deutschland stoßen diese Forderungen meistens auf schroffe Ablehnung. In den letzten Jahren wurde die deutsche Finanzpolitik vor allem von der US-Regierung und amerikanischen Ökonomen wie Larry Summers und Paul Krugman als zu restriktiv angegriffen. Der Vorwurf lautete, Ausgabensenkungen und Steuererhöhungen würden die Lage der Staatsfinanzen nur verschlimmern, weil die Konjunktur leidet. Man „spare sich kaputt“. Ein Blick auf die aktuelle Wirtschaftsentwicklung lässt allerdings Zweifel an dieser Sicht der Welt aufkommen. Die Finanzpolitik der USA selbst war sehr restriktiv - das konjunkturbereinigte Defizit wurde seit 2011 um rund $5 \%$ des BIP in den letzten Jahren reduziert. Im gleichen Zeitraum stieg das Wirtschaftswachstum von mageren 1,6\% im Jahr 2011 auf über 2\% in den Jahren danach, 2015 werden voraussichtlich über 3\% erreicht. Die Haushaltskonsolidierung hat die Erholung der US-amerikanischen Wirtschaft nicht verhindert. Eine ähnliche Entwicklung hat die britische Wirtschaft durchlaufen. Stefan Homburg hat in dieser Zeitschrift kürzlich auf die Entwicklung in Lettland, Litauen und der Slowakei hingewiesen. Das sind Beispiele für Länder, die in der letzten Wirtschaftskrise massive Austeritätsprogramme durchlaufen haben. Diese Programme waren ausnahmslos erfolgreich: Die Verschuldungsquoten sind gesunken und die Wirtschaft hat sich dort schnell erholt.

Sicherlich gibt es andere Fälle: In vielen Krisenstaaten der Eurozone sind Kürzungen im Staatshaushalt mit einem wirtschaftlichen Einbruch einhergegangen. Diese Staaten haben jedoch vor der Krise eine Phase schuldenfinanzierter Ausgabenexpansion im privaten und öffentlichen Sektor durchlaufen. Das hat zu Überschuldung und einem Verlust an preislicher Wettbewerbsfähigkeit der heimischen Wirtschaft geführt. In dieser Lage ist eine längere Anpassungsrezession unvermeidlich. Durch eine weitere Ausdehnung der öffentlichen Verschuldung lässt sich die Anpassung vielleicht verzögern, verhindern kann man sie nicht. Es ist sicherlich richtig, dass prozyklische Finanzpolitik vermieden werden sollte, solange die Verschuldungssituation des Staates dies erlaubt. Aber die These, dass wirtschaftliche Erholung und fiskalische Konsolidierung einander ausschließen, ist in dieser Allgemeinheit falsch.

Zum Abschluss des Dresdner Gipfels berichtete Bundesfinanzminister Wolfgang Schäuble, man sei sich einig gewesen, dass Strukturreformen der richtige Weg sind, um mehr Wachstum zu erreichen. Dass neue schuldenfinanzierte Ausgaben nicht in den Vordergrund gestellt wurden, heißt allerdings nicht, dass der Streit über den richtigen finanzpolitischen Weg ausgeräumt ist. Eine subtilere Variante der Argumentation für Nachfragestimulierung behauptet, dass Strukturreformen wie beispielsweise eine Lockerung des Kündigungsschutzes, kurzfristig kontraktiv wirken. Expansive Fiskalpolitik sei notwendig, um die schwierige Reformphase zu überbrücken. Der Kreativität beim Erfinden von Gründen für mehr schuldenfinanzierte Ausgaben sind keine Grenzen gesetzt. Die Debatte wird weitergehen.

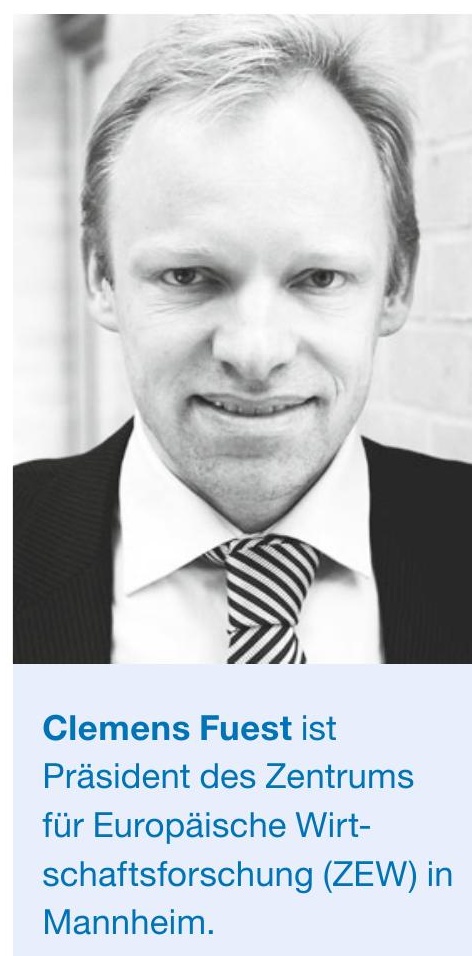

Clemens Fuest ist

Präsident des Zentrums

für Europäische Wirt-

schaftsforschung (ZEW) in

annheim 
Beim zweiten Gipfelthema, der Regulierung des Finanzsektors, gibt es unter den Finanzministern und Notenbankchefs keine so grundsätzlichen Meinungsverschiedenheiten. Dass der Finanzsektor besser beaufsichtigt werden muss und effektivere Regulierungen notwendig sind, und dass seit der Finanzkrise vieles verändert wurde, darüber besteht Einigkeit. Leider scheint keiner der G7-Staaten sonderlich beunruhigt darüber zu sein, dass grundlegende Probleme im Finanzsektor nach wie vor ungelöst sind. Die Eigenkapitalausstattung der Banken ist immer noch viel zu dünn, um im Krisenfall hinreichend Schutz zu bieten und die Steuerzahler davor zu bewahren, erneut für Bankenverluste in Anspruch genommen zu werden. Nach wie vor können Banken Staatsanleihen kaufen, ohne dafür Eigenkapital vorhalten zu müssen. Aus der Politik hört man dafür die Begründung, die Finanzierung der hohen öffentlichen Schulden wäre ohne dieses Privileg für Banken zu teuer. Das ist eine Milchmädchenrechnung. Es ist genau umgekehrt: Weil Banken ohne Eigenkapitalunterlegung in großem Umfang Staatsanleihen gehalten haben, ist die letzte Finanzkrise vor allem in der Eurozone schärfer ausgefallen und mehr Banken mussten mit Steuergeldern gerettet werden, als es bei besserer Eigenkapitalausstattung der Fall gewesen wäre. Und genau deshalb sind die Staatsschulden jetzt höher.

Das dritte Thema auf der Tagesordnung, die Steuervermeidung multinationaler Unternehmen, ist schon seit einigen Jahren Gegenstand von G7-, G8- und G20Wirtschaftsgipfeln. Die OECD wurde beauftragt, Vorschläge zur Eindämmung der Steuervermeidung zu machen. Fortschritte, die über Ankündigungen hinausgehen, sind aber bislang kaum sichtbar. Daran konnte der Dresdner Gipfel schon deshalb wenig ändern, weil wichtige Akteure fehlten. Viele kleinere Staaten, beispielsweise Irland oder die Schweiz, müssen sich an Maßnahmen gegen unerwünschte Steuergestaltungen beteiligen, sonst sind Fortschritte schwer erreichbar. Gleichzeitig saßen Regierungen am Tisch, die zwar gegen Steuervermeidung wettern, in ihren Steuersystemen aber laufend neue steuerliche Gestaltungsmöglichkeiten eröffnen. Dazu gehört beispielsweise Großbritannien. Dort wurde erst 2013 eine Patentbox eingeführt. Sie schafft Anreize, Patente aus anderen Ländern nach Großbritannien zu verlagern, weil dort Einnahmen aus der Nutzung dieser Patente nur in Höhe von $10 \%$ besteuert werden. Die USA lassen bei der Besteuerung von Auslandsgewinnen Lücken zu, die es US-amerikanischen Unternehmen erleichtern, in anderen Staaten, beispielsweise in Europa, Steuern auf Unternehmensgewinne zu vermeiden. Eine zentrale Ursache bei der Steuervermeidung durch multinationale Unternehmen liegt darin, dass viele Staaten diese Steuervermeidung aus nationalen wirtschaftlichen Interessen fördern, oft auf Kosten anderer Staaten. Hier Fortschritte zu erzielen, erfordert das Bohren dicker Bretter und die Bereitschaft der beteiligten Staaten, Interessengegensätze auszuräumen. Ein erster Schritt könnte darin liegen, dass die EU-Staaten sich vornehmen, ihre Doppelbesteuerungsabkommen mit Drittstaaten zu koordinieren. Das würde zumindest die Gewinnverlagerung in Steueroasen außerhalb der EU erschweren.

Neben dieser offiziellen Tagesordnung gab es in Dresden so etwas wie einen „Elephant in the Room“, also ein großes Problem, dass alle beschäftigt, über das aber nicht gesprochen werden sollte: Griechenland. Bei diesem Thema waren die Äußerungen im Umfeld des Treffens typisch für die übliche Gipfelrhetorik. Die USA forderten die Europäer auf, endlich zu einem „Deal“ zu kommen. Beide Seiten müssten sich bewegen. Dabei wird leicht übersehen, dass es um deutlich mehr als ein paar Milliarden Euro geht. Es geht um die Frage, ob die Mitgliedstaaten der Eurozone auf

Clemens Fuest ZEW

fuest@zew.de Dauer weiche Budgetrestriktionen haben werden, mit der Konsequenz, dass die Staatsfinanzen in Europa destabilisiert werden. Die Verhandlungen der nächsten Wochen werden zeigen, wie Europa diese Frage beantwortet. 